\title{
CABAÑAS ECOLÓGICAS: UN RECURSO PARA POTENCIAR EL DESARROLLO TURÍSTICO DE LAS COMUNIDADES RURALES
}

\author{
ECOLOGICAL CABINS: A RESOURCE TO POTENTIATE THE \\ TOURIST DEVELOPMENT OF RURAL COMMUNITIES
}

\author{
Samuel Guillén Herrera \\ Katherine León Martínez \\ Alexandra Fuentes \\ Universidad de Guayaquil, Ecuador. samuel.guillenh@ug.edu.ec
}

\section{RESUMEN}

El Turismo Comunitario, se ha convertido en un medio de desarrollo de las comunidades que lo practican, con la participación activa de sus miembros en diferentes servicios turísticos que estos ofrecen, desde la operación turística, el servicio de alimentos y bebidas, la recreación y el alojamiento, siendo en este último en el que propone a la fabricación de cabañas ecológicas como un medio de atracción para el hospedaje en las comunidades, con una visión ambientalista, de valoración del entorno y de respeto a la naturaleza, generando un desarrollo socio económico de la comunidad, con un mínimo impacto ambiental.

Palabras clave: Cabañas ecológicas, desarrollo, turístico, comunitario.

\section{ABSTRACT}

The community tourism, has become in a way of development of the communities that practice it, with the active participation of their members in the different tourist services that they offer, from the tourist operation, service of beverages and food, recreation and hospitality in the different accommodation. It is in the last service that proposes the manufacture of ecological cottages as a way of entertainment to the communities' accommodation with an environmentalist vision, valuation of the environment, and the respect of the nature, bringing a social economic development of the community, with a little bit of the environmental impact.

Keywords: Ecologic cabins, tourist, development, communities.

\section{Introducción}

Actualmente, la actividad turística y sus derivados, como el servicio de alimentos y bebidas, el entretenimiento, alojamiento, entre otros, está ampliamente extendida por todo el mundo y su influencia directa sobre la economía de ciertas regiones o países, especialmente en aquellos que se encuentran en vías de desarrollo, es muchas veces determinante para el alcance de alentadores índices de crecimiento económico y social, pero sin embargo trae como consecuencia un gran impacto ambiental.

El turismo, como actividad productiva, puede ser ampliamente beneficioso, pero también, considerablemente destructivo si no es manejado adecuadamente, ya que puede acabar con la riqueza de los patrimonios naturales y culturales de cualquier país. Esta realidad y el inminente riesgo de deterioro en los recursos, que surgen como consecuencia de diversas actividades económicas, ha provocado un fuerte movimiento internacional, nacional y local, interesado en convertir las 
prácticas tradicionales de los sectores empresariales en prácticas sostenibles.

Una de las prácticas que complementan la sostenibilidad del turismo comunitario es la hospedería comunitaria, la cual en los diferentes estudios realizados, se determina que este servicio es ofrecido por las familias de estas zonas, quienes facilitan sus viviendas para que el turista pueda alojarse, logrando un encuentro cultural, mediante el desarrollo de actividades económicas en base a la autogestión de sus habitantes. Esta actividad ha beneficiado económicamente a muchas familias en zonas de bajo nivel socio económico, pero sin embargo estas habitaciones de alquiler dentro de los hogares, ha quitado privacidad al convivir familiar, lo que lleva a buscar otras alternativas de hospedería comunitaria.

Otro de los mayores problemas no solo a las zonas donde se efectúa el turismo rural y comunitario, sino en el mundo, es la reutilización indebida de residuos de botellas plásticas que trae como consecuencia daños ambientales, estudio en esta investigación, es proponer soluciones que puedan beneficiar a las comunidades y desarrollar un turismo sostenible a través de la implementación de Cabañas Ecológicas dirigidas a la hospedería comunitaria.

La comunidad 23 de Noviembre ubicada en el Cantón Naranjal, en la Provincia del Guayas en el Ecuador, es una zona agrícola por excelencia, tierra bella rodeada de cascadas y paisajes visitada por muchos turistas, que al momento de alojarse optan por realizarlo en zonas de camping, o en habitaciones dentro de los hogares de pocas familias que ofrecen este servicio, por lo que se busca tener otras alternativas de hospedaje, que tengan servicios óptimos de calidad para que el turista tenga una grata experiencia de alojamiento y que a la vez sea amigable con el medio ambiente.

La implementación de las Cabañas Ecológicas, con las técnicas adecuadas para su construcción y su operación, ha contribuido al desarrollo social económico de la comunidad y a disminuir el impacto ambiental del sector, sumándose al inventario de servicios turísticos con una alta valoración en el análisis de la calidad de esta oferta.

\section{Antecedentes}

Existen varios referentes a nivel mundial, uno de estos, son las construcciones desarrolladas por Michael Reynolds en la década de 1970 en los Estados Unidos. Estos desarrollos eran construidos con botellas de vidrio (Reynolds, 2007). Luego, en el año 2005, se encuentra el segundo referente, edificado en Serbia por el profesor de ciencias físicas Tomislav Radovanovic. En Kragujevac, 130 kilómetros al sur de Belgrado, Radovanovic edificó una casa de $60 \mathrm{~m} 2$ sustituyendo los ladrillos por 14.000 botellas de plástico rellenas con tierra (Clarin, 2007). Posteriormente, en el 2007, se documenta un templo localizado en Tailandia, construido por monjes budistas llamado Wat $\mathrm{Pa}$ Maha Chedi Kaew en la provincia de Sisaket, unos 600 kilómetros al nordeste de Bangkok. Para su construcción se emplearon más de un millón de botellas recicladas de vidrio. Con su disciplina, los monjes buscaban crear conciencia ambiental mediante la recolección de botellas de color ámbar y verde, creando una edificación útil y estéticamente bella (País, 2005).

El templo que se encuentra en Tailandia en la provincia de Sisaket, aproximadamente a 300 millas al noroeste de Bangkok está Arquitectura Ideal, 2015). En la isla Príncipe Eduardo en Canadá se ha convertido una capilla construida de botellas en zona turística; en Bariloche encontramos casas hechas con latas recicladas. Estos ejemplos son muestras de que los materiales pueden ser usados ya que los encontramos en la basura domiciliaria. En Ecuador, la región que más recicla es la Insular o Galápagos con el $13.90 \%$ de los hogares que participan en causas Ambientales.

A nivel latinoamericano, se ha construido con esta técnica acueductos, tanques de agua, muros de cerramiento, columnas y cubiertas, tanto en viviendas como en edificios de uso comunal en varias comunidades de Honduras, Bolivia, Salvador y Colombia (Pais, 2005). La empresa que ha desarrollado estos proyectos con comunidades es Eco-Tec Soluciones Ambientales, que se estableció en Honduras, en el año 2001, con la finalidad de prestar asesorías en materia ambiental y en el manejo y aprovechamiento de residuos sólidos.

Nació queriendo dar soluciones Innovadoras y dinámicas a la problemática de la basura. El sistema de construcción con botellas desechables PET, se ha desarrollado y puesto a 
prueba desde antes de constituir la empresa con varias obras experimentales, tales como la 'Casa Ecológica' y el 'Acueducto romano en Botellas', ubicadas en el Eco parque El Zamorano situado en los alrededores de Tegucigalpa, Honduras.

La construcción de cabañas ecológicas a base de botellas plásticas recolectadas a través de una campaña que se realiza en las comunidades, Su construcción es a base de técnicas artesanales como la parcilla, adobe, entre otros, pero son igual de resistentes y de larga duración y son más baratos. Los pisos pueden ser de piedra volcánica, madera, tierra con acabado de aceite de linaza y cera de abejas. Para pintar las casas puede recurrir a la gama de colores que nos ofrecen las arcillas, plantas y flores. El corcho natural es un gran aislante térmico, acústico y no emite partículas toxicas ni vapores, no acumula energía estática, permite la transpiración de muros, absorbe la vibración, no es inflamable y se puede reciclar. Decenas de científicos y empresarios han desarrollado materiales ecológicos aprovechando desechos como botellas de plástico, vidrio, cartón reciclado y hasta fibras obtenidas de la mezclilla.

Desde una perspectiva macroeconómica la escala y sostenibilidad de la economía debe situarse dentro de los límites de la capacidad de carga de un territorio, con la finalidad de que puedan mantenerse y reproducirse las sociedades y culturas humanas, considerando un consumo adecuado de recursos naturales (Jacobs, 1996).

La optimización de los recursos naturales de una determinada zona, nos ayudará a un equilibrado desarrollo humano desde el plano individual y colectivo de sus economías, cultura e interacción con la naturaleza. En varios países latinoamericanos el turismo sostenible se empieza a evidenciar en la construcción de pequeños refugios construidos de botellas de plástico.

En países como Bolivia nace una iniciativa para ayudar a personas de escasos recursos, planteando un proyecto de casas PET que no es más que casas hechas con materiales reciclables y reutilizados para realizar estructuras como paredes, tachos de basura, entre otros, cumpliendo de esta manera con tres principios: económico, social y ambiental que promueve el ahorro y responsabilidad en el uso de los recursos y la integración de la comunidad a estos proyectos.

\section{Materiales y Métodos}

Dentro de la investigación se ha tomado en cuenta artículos que demuestran que las propuestas de turismo sustentable a través de cabañas ecológicas tienen menor impacto ambiental, así lo muestra Ruiz (2012) quien indica que las emisiones producidas por los materiales empleados en la construcción como el acero, el concreto y el ladrillo implica un alto consumo de cantidades representativas de energía, lo que a su vez contribuye al deterioro ambiental.

Ruiz plantea la necesidad de desarrollar un estudio de comportamiento técnico - mecánico, como base de análisis sismo resistente, sobre un sistema constructivo que actualmente ha empezado a tomar gran auge en el mundo, como una técnica constructiva para viviendas económicas y construcciones Post- desastre llamada construcciones con botellas PET.

Normalmente, debido al déficit de viviendas de las grandes ciudades de sectores rurales que han sido afectados por fenómenos naturales, como alternativa a este reto ha surgido una solución tecnológica llamada construcción con botellas, un sistema de autoconstrucción con botellas PET (PLÁSTICAS) no retornables de manera de ladrillos, las botellas se rellenan con tierra u otros materiales del lugar y se vinculan unas a otras por medio de tensores formando un sistema biométrico. Este sistema ha mostrado ser de bajo impacto ecológico y medioambiental (por la reutilización de botellas y tierra), de bajo costo, ya que se emplea mano de obra no calificada y materiales considerados desperdicios, proporcionando no solo viviendas, también sectores con afluencia de turistas que les llama la atención estas nuevas técnicas.

Por esta razón, el Grupo GRIME (Grupo de investigación de materiales y estructuras) documentaron el proceso constructivo y el análisis de las características básicas de los elementos que conforman el sistema, Así mismo se hicieron unas pruebas piloto de comportamiento mecánico de muros y tanque de forma que sirvan para el aporte de las necesidades de las comunidades más vulnerables.

El programa de turismo sostenible Rainforest Alliance, (2012) plantea lo siguiente: ayudar a los consumidores a identificar programas de empresas solidas en materia de turismo sostenible es decir, que debe existir principios 
mínimos de sostenibilidad a los que una empresa turística debe aspirar, la Rainforest afirma que las buenas prácticas de manejo representan una herramienta para poder cumplir con estos criterios ya que se pueden realizar negocios sin afectación mínima al medio ambiente, por ejemplo:

Servir de común denominador para que los medios de información reconozcan a los proveedores de turista sostenible.

Ofrecer a los programas gubernamentales, no - gubernamentales y privados un punto de partida para elaborar requisitos de turismo sostenible.

La alianza concibe criterios globales de turismo sostenible como el principio de un proceso para establecer la sostenibilidad como la práctica modelo en todas las formas de turismo. Proteger el medio ambiente en que se desarrolla el personal y la empresa no solo la importancia al dinero, a la sociedad personal o al medio ambiente.

Dentro de la sostenibilidad existen tres reglas básicas para aplicar la sostenibilidad en la empresa, estas son:

1. Ningún recurso renovable debe utilizarse a un ritmo superior al que se requiere para reponerlo.

2. Ningún producto contaminante debe producirse a un ritmo superior al que pueda ser reciclado, neutralizado o absorbido por el medio ambiente.

3. Ningún recurso no renovable debe aprovecharse a mayor velocidad de la manera necesaria para sustituirlo por un recurso renovable utilizado de manera sostenible.

El haber preparado y operado de manera eficiente bajo un esquema claro de visión, misión, valores y políticas en pro de una estrategia de sostenibilidad, ha sido una de las principales razones que le permitieron al hotel Villa Colonial certificarse, a finales del año 2007 como uno de los más representativos premios en sostenibilidad, como lo es el programa de certificación para turismo sostenible GREEN DEAL, obteniendo un merecido primer lugar, en cuanto al porcentaje de cumplimiento de la normativa para determinar el desempeño sostenible, utilizada por esta institución.

Por otra parte, la ONU ha alertado a los gobiernos sobre el agravamiento de los escases de vivienda en las ciudades que se presentara en los próximos 25 años. En el informe (ONUHABITAT, 2010) determinó que el $40 \%$ de la población urbana necesitara viviendas en infraestructuras de servicios básicos de aquí al 2030, el estudio advirtió que esta necesidad se sumara a la ya existente demanda de 2.000 millones de personas, que actualmente comprenden el $50 \%$ de la población del planeta, sin embargo, dar solución por parte de los gobiernos a este problema, presenta su mayor dificultad en la financiación de proyectos de bajo costo.

Los Países Bajos han logrado reciclar más del $80 \%$ de sus residuos y las empresas consideran la basura como un negocio productivo, pues la actividad de reciclar genera un beneficio social y económico a su vez, convirtiendo el proceso en una cadena de valor. Combinaciones innovadoras de buen manejo de residuos y eco diseño han aumentado la competitividad del país (Albert, 2015).

Se aplicó una metodología integrada de investigación acción descrita de la siguiente manera por el arquitecto Horacio Berretta:

"La metodología integrada de investigación acción implica la ejecución inter disciplinaria y grupal de tareas de gabinete y campo, con evaluaciones cíclicas capaces de ir generando un retorno para retro alimento de la investigación" (Berretta, 2010, pag 5).

\section{Sistema constructivo}

Se va introduciendo el material dentro de la botella a través de un embudo y se va apisonando cada capa hasta llenarla. Cuando la botella está llena no debe presentar abolladuras y al presionarla, no se debe hundir ninguna de sus caras laterales.

\section{Pega}

Existen tres tipos de mezcla para el sistema: Arena-cemento: esta mezcla se emplea en diferentes proporciones como pega 0 para pañetes en proporciones 1:4, 1:6 o 1:10, dependiendo del uso.

Cal-tierra: empleado como acabado final del sistema constructivo.

Tierra: utilizada como pega entre hiladas. 


\section{Cimentación}

Se debe tener una base de cimentación que garantice al menos la resistencia requerida para el peso propio de los muros hechos en botellas, normalmente se recomienda el uso de una placa de base en concreto ciclópeo. Sobre estos cimientos se recomienda colocar piedra laja, ladrillo tolete común o ladrillo tolete cocido con algún tipo de impermeabilizante, que aísle la construcción de la humedad por capilaridad.

\section{Muros}

Para la construcción de los muros es necesario que esté construida y nivelada la placa base, para ello se coloca la primera capa de mortero, que es una mezcla de arena-cemento. Sobre ella se colocan las botellas hasta obtener la primera hilada. La botella de la segunda hilada se coloca en el espacio medio de las botellas de la primera hilada. A medida que se van colocando las botellas se van amarrando por la cintura de la botella. Luego se procede a amarrar las tapas de las botellas, formando un amarre biométrico.

Una vez obtenida la altura requerida se procede a llenar con los escombros de tamaño medio los espacios vacíos que hay entre cada botella de manera que se nivele la superficie para proceder a colocar la primera capa de pañete.

Para su construcción se coloca una primera capa en arena-cemento y se van colocando las botellas de manera radial con la tapa hacia adentro. Las tapas deben formar un anillo simétrico en el centro, que se va amarrando a medida que se va colocando las botellas. En el centro de esta columna se colocan los refuerzos que sean necesarios para formar la columna y soportar las solicitaciones de cubierta.

Tabla 1. Resultados de Prueba y Resistencia

\begin{tabular}{|c|l|c|c|}
\hline Probeta & Relleno & Tamaño $(\mathrm{ml})$ & Carga máxima soportada (kN) \\
\hline 1 & Escombro & 600 & 310 \\
\hline 2 & Escombro & 1.500 & 50 \\
\hline 3 & Mini relleno sanitario & 2.000 & 101 \\
\hline 4 & Tierra arenosa & 600 & 70 \\
\hline 5 & Tierra arenosa & 1.500 & 5 \\
\hline 6 & Vacía & 600 & 4 \\
\hline 7 & Vacía & 1.500 & 5 \\
\hline
\end{tabular}

Nota: Recuperado de Nuevas alternativas en la construcción: Botellas PET con relleno de tierra. 2012. Daniel Ruiz

\section{Prueba de Resistencia y compresión}

Carga máxima resistente para estas primeras pruebas piloto. Se probaron botellas de tamaños de $600 \mathrm{ml}, 1.500 \mathrm{ml}$ y $2.000 \mathrm{ml}$ y con diferentes rellenos: escombro, tierra arenosa, vacías y con mini relleno sanitario. En la totalidad de los casos las botellas fueron ensayadas con las tapas plásticas roscadas en su extremo correspondiente.

De acuerdo con estas primeras pruebas, el relleno que mejor soporta la aplicación de la carga es el escombro seguido por la tierra arenosa.

\section{Conclusiones}

\section{Óptica Ambiental}

Ventajas y limitaciones de las botellas PET

1. Duración: Las botellas plásticas (PET) tienen un periodo de degradación en el medio ambiente calculado en 200 a 300 años. Con lo cual se puede garantizar, por ese periodo, la estabilidad del material que contiene la tierra.

2. Buen aislamiento térmico: por tener como relleno tierra y un espesor mayor a $28 \mathrm{~cm}$., resulta ser un buen aislamiento térmico, generando un diseño bioclimático.

3. Economía: Permite un ahorro hasta de $50 \%$ en materiales en comparación con la construcción tradicional.

4. Autoconstrucción: El proceso de construcción es realizado por la misma comunidad necesitada, sin necesidad de una capacitación particular. 
5. Botellas: No hay restricción por tamaño, forma o marca de las botellas para su uso en el sistema.

6. La mayor limitación que presenta el sistema es que no tiene reglamentación, ni estudios de caracterización, a nivel mundial y nacional, que permitan determinar el comportamiento del sistema.

\section{Óptica Económica}

La economía está en que, por su buena aislación térmica, se pueden utilizar en cerramientos con un espesor menor; y que, por su liviandad, se abarata en traslados y en cimientos.

Por otra parte, hay un "ahorro a largo plazo" por la reducción de la contaminación del medio ambiente, mediante el reciclado de materiales de descarte. El desarrollo de investigación ha demostrado que el sistema tiene características de estabilidad para ser empleado en construcciones de bajo costo y post desastre.

\section{Social}

La actividad turística convive con la belleza natural que la rodea y además con una cultura local en este caso las comunidades ya que son referentes en el mercado estas personas comparten objetivos de desarrollo, trabajar para la conservación y sostenibilidad de sus recursos naturales, el turismo cada vez es más frecuente y fortalecer a las comunidades por medio de propuestas que ayuden a incentivar que puedan trabajar en su entorno y revitalizar la economía local y ayudaría a la mejora de indicadores de calidad de vida que comparten estos actores de desarrollo que comparta la visión turística por medio del rescate y valorización de sus elementos.

Las actividades se realizarán sin perjudicar a las "comunidades", unidades, al contrario la participación e integración de los niños de la comunidad ayudará a crear una conciencia de lo que los rodea en este caso de no afectar a la naturaleza.

Igualmente, la participación a nuevos desafíos dentro de la comunidad ayudando a mejoras de desarrollo de la comunidad como una experiencia laboral de los actores locales.

\section{Referencias}

Albert, B. (5 de abril de 2015). Manejo de residuos sólidos. Obtenido de Manejo de residuos sólidos: http://preserveplanet.org/index.php? page $=$ manejoResiduos

Alliane, R. (miércoles de julio de 2012). Programa de Turismo Sostenible. Obtenido de Rainforest Alliance: www.rainforest-alliance.org.

Berretta. (2010). Aplicación de Material Plástico reciclado en elementos constructivos a base de cemento. IV encuentro nacional y Latinoamericano sobre edificaciones en comunidades sustentables. Córdoba

Carrero, A (2015) Arquitectura ideal. Esta casa está construida con miles de botellas de vidrio. www.arquitecturaideal.com

Clarin (28 de agosto de 2007). Una casa hecha solamente de botellas de plástico. pág. sección curiosidades.

Daniel Ruiz, c. I. (26 de julio de 2012). Nueva alternativa de construcción. Botellas PET rellenas con tierra, apuntes vol25 292; 303. Obtenido de botellas PET con relleno de tierra.

El Comercio (24 de Marzo 2015). Quito. El reciclaje en los hogares del país creció en los últimos cinco años. www.elcomercio.com.ec

Jacobs, M (1996). La economía verde. Icaria. Barcelona

Jama, M., \& Martin, J. (2013). Creación de hospederías comunitarias en la comuna Palmar Parroquia Colonche. Quito.

ONU (2011). Informe 2010 ONU-HABITAT. Nairobi

País, E. (08 de abril de 2005). Casa encantada armada con botellas de plástico causa sensación. El país De Cali, pág. 2.

Reynolds, M. (Jueves de marzo de 2007).how to build your own. En Reynolds, Earts ship (pág.305). Obtenido de solar survival architecture. 